\title{
Identification of biomarkers and mechanisms of diabetic cardiomyopathy using microarray data
}

\author{
Hui Li*, Xiaoyan Li*, Jian Guo, Guifu Wu, Chunping Dong, \\ Yaling Pang, Shan Gao, Yangwei Wang \\ Department of Endocrinology, Shaanxi Provincial People's Hospital, Xi'an, China
}

\begin{abstract}
Background: The study aimed to uncover the regulation mechanisms of diabetic cardiomyopathy (DCM) and provide novel prognostic biomarkers.

Methods: The dataset GSE62203 downloaded from the Gene Expression Omnibus database was utilized in the present study. After pretreatment using the Affy package, differentially expressed genes (DEGs) were identified by the limma package, followed by functional enrichment analysis and protein-protein interaction (PPI) network analysis. Furthermore, module analysis was conducted using $M C O D E$ plug-in of Cytoscape, and functional enrichment analysis was also performed for genes in the modules.
\end{abstract}

Results: A set of 560 DEGs were screened, mainly enriched in the metabolic process and cell cycle related process. Hub nodes in the PPI network were LDHA (lactate dehydrogenase A), ALDOC (aldolase C, fructose-bisphosphate) and ABCE1 (ATP Binding Cassette Subfamily E Member 1), which were also highlighted in Module 1 or Module 2 and predominantly enriched in the processes of glycolysis and ribosome biogenesis. Additionally, LDHA were linked with ALDOC in the PPI network. Besides, activating transcription factor 4 (ATF4) was prominent in Module 3; while myosin heavy chain 6 (MYH6) was highlighted in Module 4 and was mainly involved in muscle cells related biological processes.

Conclusions: Five potential biomarkers including LDHA, ALDOC, ABCE1, ATF4 and MYH6 were identified for DCM prognosis. (Cardiol J 2020; 27, 6: 807-816)

Key words: diabetic cardiomyopathy, expression profile, differential analysis, module analysis, glycolysis, ribosome biogenesis

\section{Introduction}

Type 2 diabetes mellitus (T2DM) remains a life-threatening disease worldwide with increasing incidence $[1,2]$. The predominant cause of death for T2DM patients was cardiovascular disease [3]. The diabetic cardiomyopathy (DCM) has been recognized as ventricular dysfunction in the absence of hypertension and coronary artery disease, may increase the risk of developing heart failure [4]. Moreover, DCM has been defined as a primary disease progressing into a metabolic disturbance that was mainly due to the elevation of free fatty acid (FFA) and the alteration of glucose metabolism, and would change the myocardial structure and function $[5,6]$. It was reported that the mortality of patients with DCM was $42 \%$, and the ST-segment elevation myocardial infarction (STEMI) and non-STEMI mortality in diabetic patients were $72 \%$ and $67 \%$, respectively [7]. Currently, there were no specific therapeutic interventions for this predominant complication, except a paucity of proposed drugs such as eplerenone [8]. The understanding of mechanisms on DCM progression would facilitate finding novel targets for treatment of this disease. Several mechanisms in

Address for correspondence: Yangwei Wang, MM, Department of Endocrinology, Shaanxi Provincial People's Hospital, No. 256 Youyi West Road, Xi'an, 710068, China, tel: +86-029-85251331, fax: +86-029-85236987, e-mail: yangweiwang15@hotmail.com

Received: 10.01.2017 Accepted: 3.05.2017

*Co-first authors 
charge of DCM were proposed. For instance, it was confirmed that FFA-mediated apoptosis, hypertrophy, and contractile dysfunction were the causative factors for DCM [6]. Oxidative stress was another major cause for the pathogenesis of DCM [9]. The overexpression of insulin like growth factor 1 was reported to act as an inhibitor in DCM development [10]. A more recent study elaborated molecular mechanisms that contributed to functional alterations in the diabetic heart and consequently identified several crucial advanced glycation end products (AGEs), fibrosis related genes including poly (ADP-Ribose) polymerase 1 (PARP-1), Otsuka Long Evans Tokushima fatty (OLETF) and matrix metalloproteinases 2 (MMP-2), inflammatory cytokines such as interleukin-1beta (IL-1 $\beta$ ), IL-6, tumor necrosis factor-alpha (TNF- $\alpha$ ) and transforming growth factor beta 1 (TGF- $\beta 1$ ) and altered pathways like mitogen-activated protein kinase (MAPK signaling) and TGF- $\beta$ signaling, as well as critical miRNAs (miR-143, miR-181, miR-103, miR-107 and miR-802) [11]. However, previous informative findings only partially elucidated the molecular mechanism involved in DCM, and future study for comprehensive illustrating the primary genes and the pathways for the prevention of DCM was needed.

So far, the patient-specific induced pluripotent stem cells (iPSCs) model has been applied to mimic the DCM condition and dilated cardiomyopathy, to investigate therapeutic strategies or epigenetic regulations in these diseases [12-14]. Among them, Drawnel et al. [13] used a patient-specific induced iPSC model to exhibit metabolic disorders during the progression of DCM and finally screened several remarkable molecular drugs such as W7 (calmodulin), penitrem A (sodium and potassium channel blocker) and MCBQ (PDE5 inhibitors) for the prevention of DCM. Although several gene alterations such as the elevated MYL2, MYL4 and $P L N$; and the decreased NPPA, NPPB and $A C T A 1$ were validated, the interactions among them and their functions were not interpreted, and thus lacked evidence for the prediction of potent therapeutic targets. Therefore, the expression profile GSE62203 deposited by Drawnel et al. [13] was re-analyzed to identify critical genes by extensive bioinformatical methods including differential analysis, protein-protein interaction (PPI) network and module analysis. Based on the above analyses, the aim herein was to uncover the interrelated regulation mechanisms of DCM and provide novel biomarkers for detection and prevention of DCM.

\section{Methods}

\section{Gene expression data}

A data set of the gene expression profile GSE62203 containing 4 treated samples (human iPS-derived CMs exposed to glucose, endothelin-1 and cortisol for 2 days in vitro) and 4 untreated samples (vehicle-control treated) was utilized in this study, which was deposited by Drawnel et al. [13] in the public Gene Expression Omnibus (GEO, http://www.ncbi.nlm.nih.gov/geo) database. In the Drawnel study, CMs were derived from CDI-MRB iPSCs (cellular dynamics international [CDI]). After being cultured for 2 days with conditions of $37^{\circ} \mathrm{C}$ and $7 \% \mathrm{CO}^{2}$, the plating medium for the $\mathrm{CMs}$ was changed for maturation medium (MM) for 3 days. After 3 days, the MM was exchanged for DM (MM+ glucose, endothelin and cortisol) for treated samples or MM+ vehicle control for untreated samples for another 2 days. Thus, the DCM condition was established. The platform for the expression profile was Affymetrix Human Genome U133 Plus 2.0 Array (Affymetrix Inc., Santa Clara, California, USA).

\section{Data preprocessing and differential analysis}

The Affy package in Bioconductor (http://www. bioconductor.org/packages/release/bioc/html/affy. html) [15] was employed to perform the pretreatment. The raw data were subjected to background correction, quantile data normalization and probe summarization recruiting the robust multi-array average (RMA) algorithm [16]. After obtaining the gene expression matrix, differentially expressed genes (DEGs) between the 2 kinds of samples were selected based on a t-test using linear models for microarray data (limma, http://www.bioconductor.org/packages/ /release/bioc/html/limma.html) package of Bioconductor R [17]. The cut-off values for the DEGs identification were $\mathrm{p}<0.05$ and $\mid \log _{2}$ fold change $\mid>0.5$.

\section{Functional enrichment analysis for the DEGs}

To explore the altered biological process (BP) and pathways, the DEGs were mapped into gene ontology ([GO], http://www.geneontology. org/) and Kyoto Encyclopedia of Genes and Genomes ([KEGG], http://www.genome.jp/kegg/pathway. html) databases, using Database for Annotation, Visualization and Integration Discovery ([DAVID], http://david.abcc.Ncifcrf.gov/) online tool [18] with the Modified Fisher Exact test [19]. The p-value $<0.05$ and the count (number of the genes) $>2$ were set as the threshold for significant BP terms and pathways. 


\section{Construction of PPI network}

To further explore potential correlations from the protein level, which facilitated to illustrate the underlying molecular mechanisms, identified DEGs were mapped into the Search Tool for the Retrieval of Interacting Genes/Proteins ([STRING], http:// string-db.org/) database [20]. The PPI network of protein products of the genes was established, containing pairwise interactions with required confidence (combined score) $>0.4$. A protein in the network was considered as a 'node' and the 'degree' of a node referred to the interaction pair numbers of a protein. The degree was calculated for each node using connectivity degree analysis. The 'hub' node in the network was deemed as the node with high degrees.

\section{Module analysis of the PPI network}

Functional modules of the network was extracted using the MCODE [21] plug-in of Cytoscape software with default parameters (Degree Cutoff: 2, Node Score Cutoff: 0.2, K-Core: 2, Max. Depth: 100) for selection. Subsequently, high scored modules with substantial nodes were further screened out for enrichment analysis, as described above.

\section{Results}

DEGs between treated and untreated samples

Based on the aforementioned criteria, a cohort of 560 DEGs was identified between the treated and untreated samples, consisting of 264 up-regulated genes and 296 down-regulated genes (Supplementary material 1).

\section{BPs and pathways altered in the treated sample}

After GO and KEGG enrichment analysis, the up-regulated DEGs were mainly enriched in metabolic BP terms such as generation of precursor metabolites and energy (GO: 0006091), hexose metabolic process (GO: 0019318), monosaccharide metabolic process (GO: 0005996) and glucose metabolic process (GO: 0006006); and besides response to wounding (GO: 0009611); response to organic substance (GO: 0010033), regulation of cell proliferation (GO: 0042127); while the down-regulated DEGs were significantly enriched in the processes including positive regulation of macromolecule metabolic process (GO: 0010604), cellular response to stress (GO: 0033554), and the cell control related functions such as regulation of apoptosis (GO: 0042981), regulation of programmed cell death (GO:0043067), regulation of cell death (GO:0010941), cell cycle (GO:0007049) and positive regulation of cellular biosynthetic process (GO:0031328) (Table 1).

The over-represented pathways for the upregulated DEGs were glycometabolism and proteometabolism related pathways including glycolysis/ gluconeogenesis (hsa00010), fructose and mannose metabolism (hsa00051), pentose phosphate pathway (hsa00030), starch and sucrose metabolism (hsa00500), arginine and proline metabolism (hsa00330), cysteine and methionine metabolism (hsa00270); by contrast, the prominent ones for down-regulated DEGs were aminoacyl-tRNA biosynthesis (hsa00970) and arginine, and proline metabolism (hsa00330) (Table 2).

\section{The PPI network of the DEGs}

By mapping the DEGs into the STRING database, a PPI network was established, comprising of 317 nodes and 929 interactions. As revealed in Figure 1, the remarkable nodes with high degree $(>20)$ were GAPDH (degree $=49), \mathrm{FN1}$ (degree $=$ $=30)$, LDHA (degree $=28)$, ENO1 (degree $=27)$, PGK1 (degree $=26)$, ABCE1 (degree $=25)$, SOD2 (degree = 23), PKM (degree = 23), GOT1 (degree $=22$ ), HK1 (degree $=22$ ), TPI1 (degree $=21$ ), GPI $($ degree $=21)$ and ALDOA $($ degree $=21)$.

Functional module network and the enrichment analysis for genes in the modules

According to module analysis of the PPI network, four modules with a high score $(>3)$ were extracted from the PPI network. There were 14 up-regulated nodes such as ALDOC, LDHA, PGK1 and TPI1 in Module 1 with a final score of 12.923; and 10 nodes including ABCE1, GAR1 and FBL in Module 2 with a final score of 8.222. The Module 3 contained five down-regulated nodes as DDIF3, ATF4, CEBPG, CEBPB and HERPUD1 and achieved a score of 4 , while Module 4 consisted of 15 nodes such as CASQ2, CKMT2, IARS, CCT5, ACTA1, CKMT2 and MYH6 and had a score of 3.857 (Fig. 2).

The BP functions of the genes (which encode proteins in the modules) in the four modules were further analyzed. As presented in Table 3, the over-represented BPs for genes in Module 1 were predominantly correlated with the catabolic process of various carbohydrates such as glycolysis (GO:0006096), glucose catabolic process (GO:0006007), monosaccharide catabolic process (GO:0046365) and alcohol catabolic process (GO:0046164); while that for genes in Module 2 were mainly related to ribosome biogenesis and 
Table 1. Biological processes significantly affected by the DEGs in treated samples. (Top ten, ranked by gene numbers enriched in a specific process).

\begin{tabular}{|c|c|c|c|c|}
\hline Category & Term & Description & Count & $\mathbf{P}$ \\
\hline \multicolumn{5}{|c|}{ Up-regulated DEGs } \\
\hline $\mathrm{BP}$ & GO:0006091 & Generation of precursor metabolites and energy & 28 & 1.37E-13 \\
\hline BP & GO:0055114 & Oxidation reduction & 25 & $2.72 \mathrm{E}-05$ \\
\hline BP & GO:0009611 & Response to wounding & 24 & $3.98 \mathrm{E}-06$ \\
\hline $\mathrm{BP}$ & GO:0010033 & Response to organic substance & 23 & 1.07E-03 \\
\hline BP & GO:0019318 & Hexose metabolic process & 22 & 8.75E-13 \\
\hline $\mathrm{BP}$ & GO:0005996 & Monosaccharide metabolic process & 22 & $1.47 \mathrm{E}-11$ \\
\hline $\mathrm{BP}$ & GO:0042592 & Homeostatic process & 22 & $3.95 \mathrm{E}-03$ \\
\hline $\mathrm{BP}$ & GO:0006006 & Glucose metabolic process & 21 & $1.05 \mathrm{E}-13$ \\
\hline BP & GO:0042127 & Regulation of cell proliferation & 19 & 4.54E-02 \\
\hline BP & GO:0044057 & Regulation of system process & 16 & $5.92 \mathrm{E}-05$ \\
\hline \multicolumn{5}{|c|}{ Down-regulated DEGs } \\
\hline $\mathrm{BP}$ & GO:0010604 & Positive regulation of macromolecule metabolic process & 22 & $1.20 \mathrm{E}-02$ \\
\hline BP & GO:0033554 & Cellular response to stress & 21 & 1.97E-04 \\
\hline $\mathrm{BP}$ & GO:0042981 & Regulation of apoptosis & 20 & $2.32 \mathrm{E}-02$ \\
\hline BP & GO:0043067 & Regulation of programmed cell death & 20 & 2.54E-02 \\
\hline BP & GO:0010941 & Regulation of cell death & 20 & $2.62 \mathrm{E}-02$ \\
\hline BP & GO:0009891 & Positive regulation of biosynthetic process & 19 & 1.17E-02 \\
\hline BP & GO:0007049 & Cell cycle & 19 & 3.14E-02 \\
\hline BP & GO:0006412 & Translation & 18 & $6.09 \mathrm{E}-06$ \\
\hline BP & GO:0006396 & RNA processing & 18 & 2.41E-03 \\
\hline $\mathrm{BP}$ & GO:0031328 & Positive regulation of cellular biosynthetic process & 18 & 2.05E-02 \\
\hline
\end{tabular}

DEG - differentially expressed genes; BP — biological process; GO - gene oncology; Count - gene numbers enriched in a specific BP term.

Table 2. Pathways significantly altered by the DEGs in treated samples.

\begin{tabular}{|c|c|c|c|c|}
\hline Category & Term & Description & Count & $\mathbf{P}$ \\
\hline \multicolumn{5}{|c|}{ Up-regulated DEGs } \\
\hline KEGG & hsa00010 & Glycolysis / Gluconeogenesis & 15 & $3.66 \mathrm{E}-12$ \\
\hline KEGG & hsa00051 & Fructose and mannose metabolism & 6 & 4.49E-04 \\
\hline KEGG & hsa00330 & Arginine and proline metabolism & 7 & $5.12 \mathrm{E}-04$ \\
\hline KEGG & hsa00500 & Starch and sucrose metabolism & 6 & $1.21 \mathrm{E}-03$ \\
\hline KEGG & hsa00030 & Pentose phosphate pathway & 5 & $1.25 \mathrm{E}-03$ \\
\hline KEGG & hsa04810 & Regulation of actin cytoskeleton & 12 & 2.84E-03 \\
\hline KEGG & hsa04510 & Focal adhesion & 10 & 1.57E-02 \\
\hline KEGG & hsa00270 & Cysteine and methionine metabolism & 4 & 2.77E-02 \\
\hline KEGG & hsa05012 & Parkinson's disease & 7 & $3.78 \mathrm{E}-02$ \\
\hline KEGG & hsa04610 & Complement and coagulation cascades & 5 & 4.46E-02 \\
\hline KEGG & hsa05211 & Renal cell carcinoma & 5 & 4.67E-02 \\
\hline \multicolumn{5}{|c|}{ Down-regulated DEGs } \\
\hline KEGG & hsa00970 & Aminoacyl-tRNA biosynthesis & 7 & 2.92E-05 \\
\hline KEGG & hsa00330 & Arginine and proline metabolism & 4 & 4.56E-02 \\
\hline
\end{tabular}

DEG — differentially expressed genes; KEGG — Kyoto Encyclopedia of Genes and Genomes; Count — gene numbers enriched in a specific biological process term 


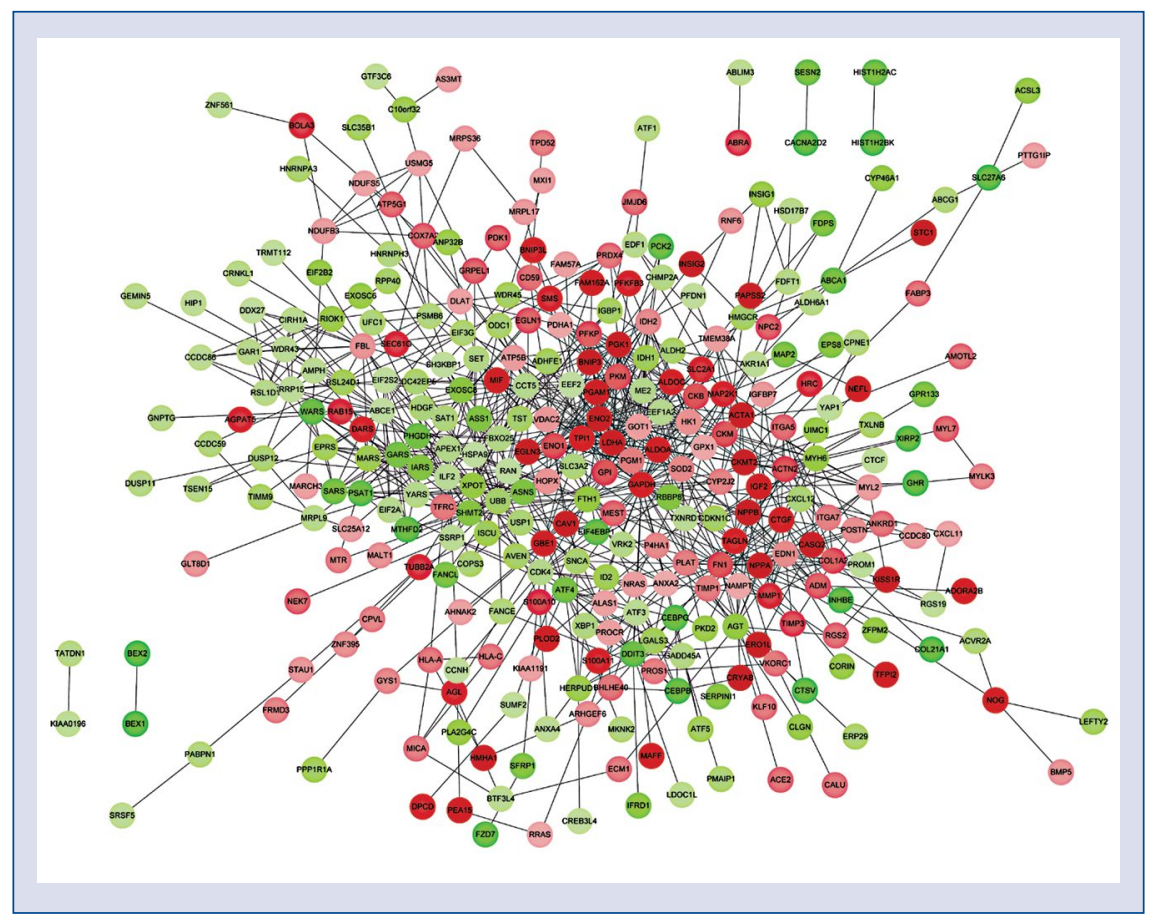

Figure 1. Protein-protein interaction network of differentially expressed genes in iPS-derived cardiomyocytes treated by glucose, endothelin-1 and cortisol. Circles represent protein products of differentially expressed genes, and red denotes up-regulated, green denotes down-regulated; color depth indicates the significance of differential expressed genes.

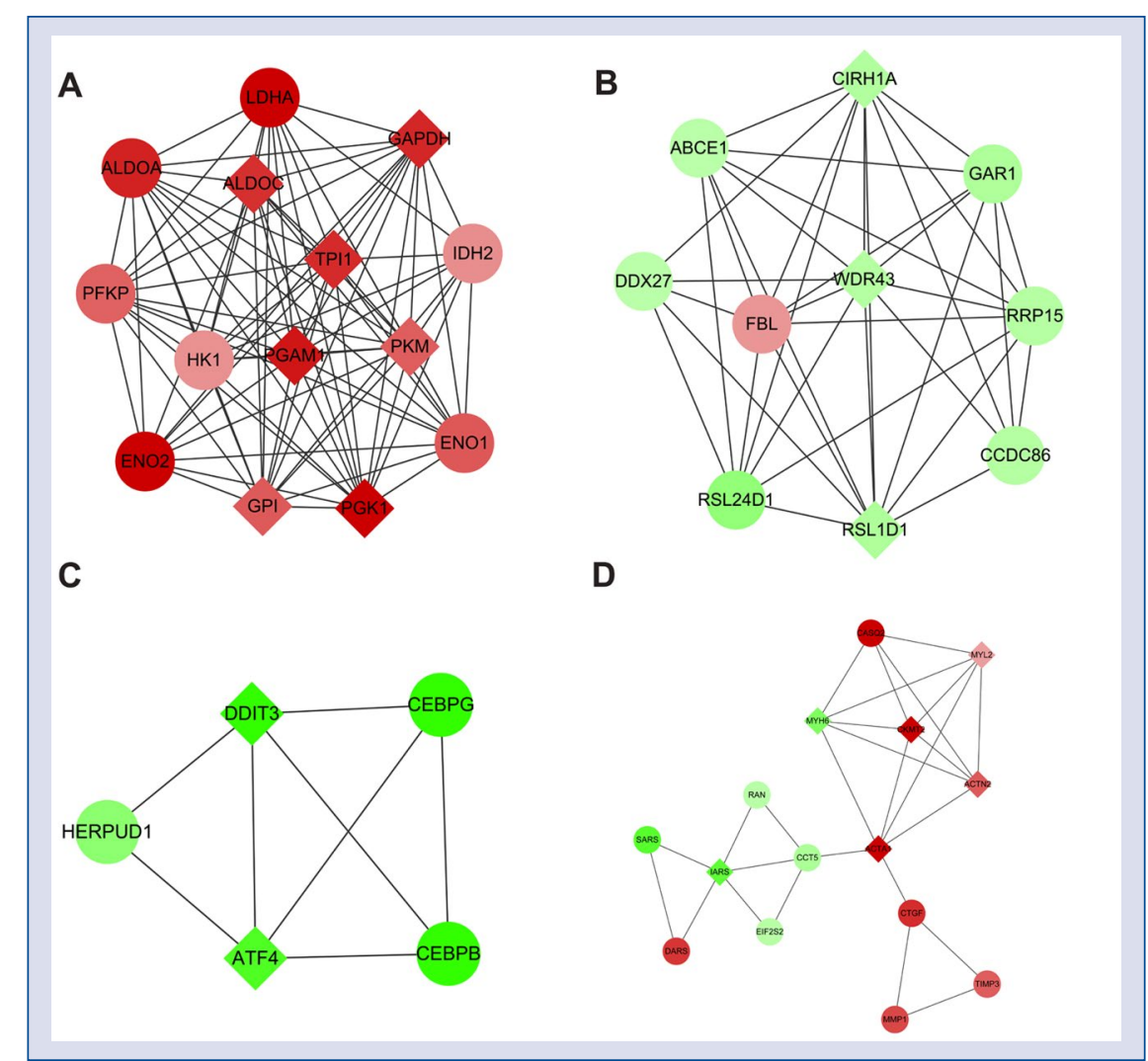

Figure 2. Modules of the protein-protein interaction network. A. Module 1; B. Module 2; C. Module 3; D. Module 4. Circles represent protein products of differentially expressed genes, and red denotes up-regulated genes, green denotes down-regulated genes, as well as diamonds stand for hub nodes; color depth indicates the significance of differentially expressed genes. 
Table 3. Significantly enriched processes of genes in the module network.

\begin{tabular}{|c|c|c|c|c|}
\hline Category & Term & Description & Count & $\mathbf{P}$ \\
\hline \multicolumn{5}{|l|}{ Module 1} \\
\hline $\mathrm{BP}$ & GO:0006096 & Glycolysis & 13 & $8.68 \mathrm{E}-30$ \\
\hline BP & GO:0006007 & Glucose catabolic process & 13 & $1.48 \mathrm{E}-28$ \\
\hline BP & GO:0019320 & Hexose catabolic process & 13 & $1.46 \mathrm{E}-27$ \\
\hline BP & GO:0046365 & Monosaccharide catabolic process & 13 & 2.12E-27 \\
\hline $\mathrm{BP}$ & GO:0046164 & Alcohol catabolic process & 13 & 1.17E-26 \\
\hline $\mathrm{BP}$ & GO:0044275 & Cellular carbohydrate catabolic process & 13 & $2.18 \mathrm{E}-26$ \\
\hline BP & GO:0016052 & Carbohydrate catabolic process & 13 & $5.18 \mathrm{E}-25$ \\
\hline BP & GO:0006006 & Glucose metabolic process & 13 & $3.64 \mathrm{E}-23$ \\
\hline BP & GO:0006091 & Generation of precursor metabolites and energy & 14 & 4.25E-22 \\
\hline BP & GO:0019318 & Hexose metabolic process & 13 & $6.06 \mathrm{E}-22$ \\
\hline \multicolumn{5}{|l|}{ Module 2} \\
\hline BP & GO:0042254 & Ribosome biogenesis & 3 & 4.78E-04 \\
\hline BP & GO:0022613 & Ribonucleoprotein complex biogenesis & 3 & 1.04E-03 \\
\hline BP & GO:0006396 & RNA processing & 3 & 9.27E-03 \\
\hline BP & GO:0006364 & rRNA processing & 2 & 2.69E-02 \\
\hline $\mathrm{BP}$ & GO:0016072 & rRNA metabolic process & 2 & $2.81 \mathrm{E}-02$ \\
\hline \multicolumn{5}{|l|}{ Module 3} \\
\hline $\mathrm{BP}$ & GO:0034976 & Response to endoplasmic reticulum stress & 3 & 3.67E-05 \\
\hline BP & GO:0045935 & $\begin{array}{l}\text { Positive regulation of nucleobase, nucleoside, } \\
\text { nucleotide and nucleic acid metabolic process }\end{array}$ & 4 & 3.77E-04 \\
\hline BP & GO:0051173 & Positive regulation of nitrogen compound metabolic process & 4 & 4.14E-04 \\
\hline BP & GO:0010557 & Positive regulation of macromolecule biosynthetic process & 4 & 4.34E-04 \\
\hline BP & GO:0031328 & Positive regulation of cellular biosynthetic process & 4 & $4.98 \mathrm{E}-04$ \\
\hline BP & GO:0009891 & Positive regulation of biosynthetic process & 4 & 5.19E-04 \\
\hline BP & GO:0042981 & Regulation of apoptosis & 4 & 8.00E-04 \\
\hline BP & GO:0043067 & Regulation of programmed cell death & 4 & 8.23E-04 \\
\hline $\mathrm{BP}$ & GO:0010941 & Regulation of cell death & 4 & $8.32 \mathrm{E}-04$ \\
\hline BP & GO:0010604 & Positive regulation of macromolecule metabolic process & 4 & $9.66 \mathrm{E}-04$ \\
\hline \multicolumn{5}{|l|}{ Module 4} \\
\hline $\mathrm{BP}$ & GO:0006936 & Muscle contraction & 5 & $1.44 \mathrm{E}-05$ \\
\hline BP & GO:0003012 & Muscle system process & 5 & $2.08 \mathrm{E}-05$ \\
\hline $\mathrm{BP}$ & GO:0030239 & Myofibril assembly & 3 & 2.07E-04 \\
\hline BP & GO:0031032 & Actomyosin structure organization & 3 & 3.70E-04 \\
\hline BP & GO:0010927 & Cellular component assembly involved in morphogenesis & 3 & $6.14 \mathrm{E}-04$ \\
\hline BP & GO:0006418 & tRNA aminoacylation for protein translation & 3 & $1.00 \mathrm{E}-03$ \\
\hline BP & GO:0043039 & tRNA aminoacylation & 3 & $1.00 \mathrm{E}-03$ \\
\hline $\mathrm{BP}$ & GO:0043038 & Amino acid activation & 3 & $1.00 \mathrm{E}-03$ \\
\hline $\mathrm{BP}$ & GO:0055002 & Striated muscle cell development & 3 & $1.28 \mathrm{E}-03$ \\
\hline $\mathrm{BP}$ & GO:0055001 & Muscle cell development & 3 & $1.48 \mathrm{E}-03$ \\
\hline
\end{tabular}

BP — biological process; GO - gene oncology; Count - gene numbers enriched in a specific BP term

processing functions including ribosome biogenesis (GO:0042254), RNA processing (GO:0006396) and rRNA metabolic process (GO:0016072). Genes in the Module 3 were significantly correlated with the metabolic process involved in the cellular biosynthesis such as positive regulation of nucleobase, nucleoside, nucleotide and nucleic acid metabolic process (GO:0045935), positive 
regulation of nitrogen compound metabolic process (GO:0051173), positive regulation of cellular biosynthetic process (GO:0031328); and besides the cell control related BPs including regulation of apoptosis (GO:0042981) and regulation of programmed cell death (GO:0043067); whereas the prominent BPs for the genes in the Module 4 were involved in the processes relating to muscle cells such as muscle contraction (GO:0006936), muscle system process (GO:0003012) and muscle cell development (GO:0055001).

\section{Discussion}

The DCM is defined as ventricular dysfunction that occurs in diabetic patients [22] and the iPSC model was applied to detect the metabolic alterations and screen potential genes and molecular drugs $[13,23]$. In the present study, the expression profile GSE62203 was utilized to conduct a series of bioinformatic analyses and as a result, identify a cohort of 560 DEGs between treated and untreated samples. The hub nodes in the PPI network were LDHA, ALDOC and ABCE1, which were also highlighted in Module 1 or Module 2 and predominantly enriched in the glycolysis and ribosome biogenesis. Besides, ATF4 was prominent in Module 3; while MYH6 was highlighted in Module 4 which was mainly involved in muscle cells related BPs.

The LDHA (lactate dehydrogenase A) is one of the subunits of LDH which play significant roles in the final step of anaerobic glycolysis by interconversion of pyruvate and lactate using $\mathrm{NADH} / \mathrm{NAD}+$ as a co-substrate to allow continuous energy production [24]. It was reported that overexpression of LDHA activity may influence normal glucose metabolism and insulin secretion in the islet beta-cell type, and also result in insulin secretory defects in some forms of T2DM $[25,26]$. In addition, the overexpression of LDHA activity might increase the lactate level and lactate-pyruvate interconversion rates in diabetes patients [27]. Similarly, the increased level of LDH was observed in the diabetic group, while luteolin exerted a protective effect against DCM by reducing the content of LDH in serum [28]. Hypoxia-inducible factor (HIF)-1, was a crucial transcription factor in brain ischemic pre-conditioning [29] and the expression of $H I F-1 \alpha$ was decreased by a diabetic environment [30]. Partial deficiency of HIF- $1 \alpha$ was proposed to increase the risk of DCM, and interestingly, $L D H A$ was one of the target genes of HIF-1 that is involved in glucose metabolism and was upregulated in the HIF- $1 \alpha$ heterozygous-null mutants [31]. In the present study, LDHA was the striking node in both PPI network and Module 1, and significantly enriched in glycolysis, giving potent evidence that $L D H A$ might emerge as a central regulator in the progression of DCM via disturbing the glycolysis process.

$A L D O C$ (aldolase $\mathrm{C}$, fructose-bisphosphate) encodes a member of the class I fructose-biphosphate aldolase family gene, which acts as a catalyst that catalyzes the reversible aldol cleavage of fructose-1,6-biphosphate and fructose 1-phosphate to dihydroxyacetone phosphate and either glyceraldehyde-3-phosphate or glyceraldehyde, respectively in the glycolysis process [32]. Increased glucose was one hallmark of diabetes mellitus (DM) and $A L D O C$ was one of the enzymes that promoted glycolysis and was induced by the elevated glucose [33]. Then, the up-regulated $A L D O C$ was a positive correlation with the increase of FFA in plasma which might impair insulin secretion to develop T2DM [34, 35]. Additionally, $A L D O C$ was up-regulated in the heart tissue in a rodent model of myocardial I/R injury [36]. Though no direct evidence existed that $A L D O C$ and $L D H A$ were interplayed with regard to diabetes or cardiomyopathy, it was indicated that $A L D O C$ and $L D H A$ were both up-regulated in a cervical cancer cell line of paclitaxel-resistant HeLa sublines [37]. On the other hand, DM was tightly related to the risk of various cancers including cervical cancer [38]. Notably, $A L D O C$ and $L D H A$ were both linked to HIF-1, which was associated with the risk of DCM as mentioned above [31]. These findings collectively suggested that the interacted $A L D O C$ and $L D H A$ might be involved in the regulation of the glycolysis process during DCM progression, as predicted by the current module analysis and enrichment analysis. However, more validations are needed to confirm the regulatory relationship between the two genes.

The $A B C E 1$ encoded ATP Binding Cassette Subfamily E Member 1 which belongs to a family member of the ATP-binding cassette (ABC) transporters and is primarily known as RNase $\mathrm{L}$ inhibitor (RLI) [39]. Zeng et al. [40] had indicated that RNase $\mathrm{L}$ activation was responsible for type I diabetes, and it was also suggested that the increased expression of RNase L or down-regulated of its inhibitor (RLI) might enhance the insulin response in muscle cells of obese people [41]. Additionally, further studies demonstrated that the mutation of $A B C B$ and gene polymorphisms of $A B C G 8$ and $A B C G 5$ have been linked to T2DM 
[42, 43]. Moreover, ABC transporters are energydependent when transporting various molecules across the biological membranes, while HF is the consequence of insufficient energy supplement of the cardiac pump. Therefore, it was hypothesized that the expression alterations of $\mathrm{ABC}$ transporters occurred during human $\mathrm{HF}[44,45]$. Due to $A B C E 1$ is a member of $\mathrm{ABC}$ transporters, and the present results indicated that the $\mathrm{ABCE} 1$ was a prominent down-regulated node in Module 2. Thus, it was predicted that the defective $A B C E 1$ might have a significant influence on the progression of DCM. However, there is no direct evidence to prove that $A B C E 1$ had interplayed with DCM, and it still needs further validation to confirm the relationship between the $A B C E 1$ and DCM.

The endoplasmic reticulum (ER) is a cell system consisting of the lipid synthesis, calcium homeostasis, protein folding, and maturation. The ER stress has been reported in the development of DCM [47, 48]. Moreover, it has been confirmed that ER-triggered apoptosis would contribute to the pathology of DCM [49]. Activating transcription factor 4 (ATF4) is a DNA binding protein. The glucagon-like peptide- 1 analog liraglutide (LIRA) was confirmed to protect against DCM by inactivating the ER stress pathway, meanwhile the expression of ATF4 was decreased with the treatment of LIRA [50], implying that ATF4 might play significant roles in the progression of DCM, as predicted in the present result that ATF4 was a prominent node in Module 3.

The cardiac muscle myosin MYH6 was decreased in type 2 Zucker diabetic fatty rats [51]. Strikingly, MYH6 was diminished under the hypertrophic stress (DM-treated with CMs) [13] and was considered a cardiac marker by fluorescent immunostaining [52]. The current results indicated that MYH6 was highlighted in Module 4 and correlated with muscle cells related BPs, suggesting that MYH6 might also be used as a biomarker for the prognosis of DCM.

\section{Conclusions}

In conclusion, five potential biomarkers including $L D H A, A L D O C, A B C E 1, A T F 4$ and $M Y H 6$ were identified for DCM prognosis. During DCM progression, $L D H A$ and $A L D O C$ might have interplayed and play significant roles via regulation of the glycolysis process. However, these findings need to be further confirmed via extensive validation.

\section{Acknowledgements}

This work was supported by the National Natural Science Foundation of China (No.81100208).

\section{Conflict of interest: None declared}

\section{References}

1. Sharma M, Nazareth I, Petersen I. Trends in incidence, prevalence and prescribing in type 2 diabetes mellitus between 2000 and 2013 in primary care: a retrospective cohort study. BMJ Open. 2016; 6(1): e010210, doi: 10.1136/bmjopen-2015-010210, indexed in Pubmed: 26769791.

2. Heather LC, Clarke K. Metabolism, hypoxia and the diabetic heart. J Mol Cell Cardiol. 2011; 50(4): 598-605, doi: 10.1016/j. yjmcc.2011.01.007, indexed in Pubmed: 21262230.

3. Scirica B, Bhatt D, Braunwald E, et al. Saxagliptin and Cardiovascular Outcomes in Patients with Type 2 Diabetes Mellitus. N Engl J Med. 2013; 369(14): 1317-1326, doi: 10.1056/nejmoa1307684.

4. Acar E, Ural D, Bildirici U, et al. Diabetic cardiomyopathy. Anadolu Kardiyol Derg. 2011; 11(8): 732-737, doi: 10.5152/akd.2011.196, indexed in Pubmed: 22137942.

5. Asghar O, Al-Sunni A, Khavandi K, et al. Diabetic cardiomyopathy. Clin Sci (Lond). 2009; 116(10): 741-760, doi: 10.1042/ /CS20080500, indexed in Pubmed: 19364331.

6. Chavali V, Tyagi SC, Mishra PK. Predictors and prevention of diabetic cardiomyopathy. Diabetes Metab Syndr Obes. 2013; 6: 151-160, doi: 10.2147/DMSO.S30968, indexed in Pubmed: 23610527.

7. Alabas OA, Hall M, Dondo TB, et al. Long-term excess mortality associated with diabetes following acute myocardial infarction: a population-based cohort study. J Epidemiol Community Health. 2017; 71(1): 25-32, doi: 10.1136/jech-2016-207402, indexed in Pubmed: 27307468.

8. Leung M, Wong VW, Heritier S, et al. Rationale and design of a randomized trial on the impact of aldosterone antagonism on cardiac structure and function in diabetic cardiomyopathy. Cardiovasc Diabetol. 2013; 12: 139, doi: 10.1186/1475-2840-12-139, indexed in Pubmed: 24083804.

9. Liu Q, Wang S, Cai Lu. Diabetic cardiomyopathy and its mechanisms: Role of oxidative stress and damage. J Diabetes Investig. 2014; 5(6): 623-634, doi: 10.1111/jdi.12250, indexed in Pubmed: 25422760 .

10. Kajstura J, Fiordaliso F, Andreoli AM, et al. IGF-1 overexpression inhibits the development of diabetic cardiomyopathy and angiotensin II-mediated oxidative stress. Diabetes. 2001; 50(6): 1414-1424, indexed in Pubmed: 11375343.

11. Bugger $\mathrm{H}$, Abel E. Molecular mechanisms of diabetic cardiomyopathy. Diabetologia. 2014; 57(4): 660-671, doi: 10.1007/s00125-014-3171-6.

12. $\mathrm{Wu} \mathrm{H}$, Lee J, Vincent LG, et al. Epigenetic regulation of phosphodiesterases $2 \mathrm{~A}$ and $3 \mathrm{~A}$ underlies compromised $\beta$-adrenergic signaling in an iPSC model of dilated cardiomyopathy. Cell Stem Cell. 2015; 17(1): 89-100, doi: 10.1016/j.stem.2015.04.020, indexed in Pubmed: 26095046.

13. Drawnel FM, Boccardo S, Prummer M, et al. Disease modeling and phenotypic drug screening for diabetic cardiomyopathy using human induced pluripotent stem cells. Cell Rep. 2014; 9(3): 810-821, doi: 10.1016/j.celrep.2014.09.055, indexed in Pubmed: 25437537. 
14. Churko JM, Sallam KI, Matsa E, et al. Epigenetic regulation of phosphodiesterases 2a and3aunderliescompromisedb-adrenergicsignaling in an ipsc model of dilated cardiomyopathy. Cell Stem Cell. 2015; 17: 1-12.

15. Gautier L, Cope L, Bolstad BM, et al. Affy-analysis of Affymetrix GeneChip data at the probe level. Bioinformatics. 2004; 20(3): 307-315, doi: 10.1093/bioinformatics/btg405, indexed in Pubmed: 14960456.

16. Horiuchi Y, Kano SI, Ishizuka K, et al. Olfactory cells via nasal biopsy reflect the developing brain in gene expression profiles: utility and limitation of the surrogate tissues in research for brain disorders. Neurosci Res. 2013; 77(4): 247-250, doi: 10.1016/j. neures.2013.09.010, indexed in Pubmed: 24120685.

17. Smyth GK. Limma: Linear models for microarray data; Bioinformatics and computational biology solutions using $r$ and bioconductor. Springer. 2005: 397, doi: 420.

18. Huang DaW, Sherman BT, Lempicki RA. Systematic and integrative analysis of large gene lists using DAVID bioinformatics resources. Nat Protoc. 2009; 4(1): 44-57, doi: 10.1038/ /nprot.2008.211, indexed in Pubmed: 19131956.

19. Grossmann S, Bauer S, Robinson PN, et al. Improved detection of overrepresentation of Gene-Ontology annotations with parent child analysis. Bioinformatics. 2007; 23(22): 3024-3031, doi: 10.1093/ /bioinformatics/btm440, indexed in Pubmed: 17848398.

20. Szklarczyk D, Franceschini A, Kuhn M, et al. The STRING database in 2011: functional interaction networks of proteins, globally integrated and scored. Nucleic Acids Res. 2011; 39(Database issue): D561-D568, doi: 10.1093/nar/gkq973, indexed in Pubmed: 21045058.

21. Rhrissorrakrai K, Gunsalus KC, Rhrissorrakrai K, et al. MINE: Module Identification in Networks. BMC Bioinformatics. 2011; 12: 192, doi: 10.1186/1471-2105-12-192, indexed in Pubmed: 21605434 .

22. Falcão-Pires I, Leite-Moreira AF. Diabetic cardiomyopathy: understanding the molecular and cellular basis to progress in diagnosis and treatment. Heart Fail Rev. 2012; 17(3): 325-344, doi: 10.1007/s10741-011-9257-z, indexed in Pubmed: 21626163.

23. Choi SuMi, Kim Y, Shim JS, et al. Efficient drug screening and gene correction for treating liver disease using patient-specific stem cells. Hepatology. 2013; 57(6): 2458-2468, doi: 10.1002/ hep.26237, indexed in Pubmed: 23325555.

24. Kolappan S, Shen DL, Mosi R, et al. Structures of lactate dehydrogenase A (LDHA) in apo, ternary and inhibitor-bound forms. Acta Crystallogr D Biol Crystallogr. 2015; 71(Pt 2): 185-195, doi: 10.1107/S1399004714024791, indexed in Pubmed: 25664730 .

25. Ainscow EK, Zhao C, Rutter GA. Acute overexpression of lactate dehydrogenase-A perturbs beta-cell mitochondrial metabolism and insulin secretion. Diabetes. 2000; 49(7): 1149-1155, indexed in Pubmed: 10909972.

26. Chen Y, Wang X, Shao X. A combination of human embryonic stem cell-derived pancreatic endoderm transplant with ldha-repressing miRNA can attenuate high-fat diet induced type II diabetes in mice. J Diabetes Res. 2015; 2015: 796912, doi: 10.1155/2015/796912, indexed in Pubmed: 26770982.

27. Avogaro A, Toffolo G, Miola M, et al. Intracellular lactate- and pyruvate-interconversion rates are increased in muscle tissue of non-insulin-dependent diabetic individuals. J Clin Invest. 1996; 98(1): 108-115, doi: 10.1172/JCI118754, indexed in Pubmed: 8690781.
28. Wang G, Li W, Lu X, et al. Luteolin ameliorates cardiac failure in type I diabetic cardiomyopathy. J Diabetes Complications. 2012; 26(4): 259-265, doi: 10.1016/j.jdiacomp.2012.04.007, indexed in Pubmed: 22626874.

29. Scornavacca G, Gesuete R, Orsini F, et al. Proteomic analysis of mouse brain cortex identifies metabolic down-regulation as a general feature of ischemic pre-conditioning. J Neurochem. 2012; 122(6): 1219-1229, doi: 10.1111/j.1471-4159.2012.07874.x, indexed in Pubmed: 22804628.

30. Thangarajah H, Yao D, Chang EI, et al. The molecular basis for impaired hypoxia-induced VEGF expression in diabetic tissues. Proc Natl Acad Sci U S A. 2009; 106(32): 13505-13510, doi: 10.1073/pnas.0906670106, indexed in Pubmed: 19666581.

31. Bohuslavova R, Kolar F, Sedmera D, et al. Partial deficiency of HIF-1 $\alpha$ stimulates pathological cardiac changes in streptozotocin-induced diabetic mice. BMC Endocr Disord. 2014; 14: 11, doi: 10.1186/1472-6823-14-11, indexed in Pubmed: 24502509.

32. Wang CF, Yuan CZ, Wang SH, et al. Differential gene expression of aldolase $\mathrm{C}$ (ALDOC) and hypoxic adaptation in chickens. Anim Genet. 2007; 38(3): 203-210, doi: 10.1111/j.13652052.2007.01605.x, indexed in Pubmed: 17539972.

33. Kim ES, Isoda F, Kurland I, et al. Glucose-induced metabolic memory in Schwann cells: prevention by PPAR agonists. Endocrinology. 2013; 154(9): 3054-3066, doi: 10.1210/en.2013-1097, indexed in Pubmed: 23709088.

34. Camps SG, Verhoef SPM, Roumans N, et al. Weight loss-induced changes in adipose tissue proteins associated with fatty acid and glucose metabolism correlate with adaptations in energy expenditure. Nutr Metab (Lond). 2015; 12: 37, doi: 10.1186/ s12986-015-0034-1, indexed in Pubmed: 26500687.

35. Kashyap S, Belfort R, Gastaldelli A, et al. A sustained increase in plasma free fatty acids impairs insulin secretion in nondiabetic subjects genetically predisposed to develop type 2 diabetes. Diabetes. 2003; 52(10): 2461-2474, indexed in Pubmed: 14514628.

36. Bao W, Aravindhan K, Alsaid H, et al. Albiglutide, a long lasting glucagon-like peptide-1 analog, protects the rat heart against ischemia/reperfusion injury: evidence for improving cardiac metabolic efficiency. PLoS One. 2011; 6(8): e23570, doi: 10.1371/ journal.pone.0023570, indexed in Pubmed: 21887274.

37. Peng X, Gong F, Chen Y, et al. Autophagy promotes paclitaxel resistance of cervical cancer cells: involvement of Warburg effect activated hypoxia-induced factor $1-\alpha$-mediated signaling. Cell Death Dis. 2014; 5: e1367, doi: 10.1038/cddis.2014.297, indexed in Pubmed: 25118927.

38. Szablewski L. Diabetes mellitus: influences on cancer risk. Diabetes Metab Res Rev. 2014; 30(7): 543-553, doi: 10.1002/ dmrr.2573, indexed in Pubmed: 25044584.

39. Chen ZQ, Dong J, Ishimura A, et al. The essential vertebrate ABCE1 protein interacts with eukaryotic initiation factors. J Biol Chem. 2006; 281(11): 7452-7457, doi: 10.1074/jbc.M510603200, indexed in Pubmed: 16421098.

40. Zeng C, Yi X, Zipris D, et al. RNase L contributes to experimentally induced type 1 diabetes onset in mice. J Endocrinol. 2014; 223(3): 277-287, doi: 10.1530/JOE-14-0509, indexed in Pubmed: 25287058.

41. Fabre O, Breuker C, Amouzou C, et al. Defects in TLR3 expression and RNase L activation lead to decreased MnSOD expression and insulin resistance in muscle cells of obese people. Cell Death Dis. 2014; 5: e1136, doi: 10.1038/cddis.2014.104, indexed in Pubmed: 24651439. 
42. Vasiliou V, Vasiliou K, Nebert DW. Human ATP-binding cassette (ABC) transporter family. Hum Genomics. 2009; 3(3): 281-290, indexed in Pubmed: 19403462.

43. Gok O, Karaali ZE, Acar L, et al. ABCG5 and ABCG8 gene polymorphisms in type 2 diabetes mellitus in the Turkish population. Can J Diabetes. 2015; 39(5): 405-410, doi: 10.1016/j. jcjd.2015.04.004, indexed in Pubmed: 26088706.

44. Ventura-Clapier R, Garnier A, Veksler V. Energy metabolism in heart failure. J Physiology. 2004; 555(1): 1-13, doi: 10.1113/ jphysiol.2003.055095.

45. Solbach TF, Paulus B, Weyand M, et al. ATP-binding cassette transporters in human heart failure. Naunyn Schmiedebergs Arch Pharmacol. 2008; 377(3): 231-243, doi: 10.1007/s00210008-0279-6, indexed in Pubmed: 18392808.

46. Licht A, Schneider E. ATP binding cassette systems: structures, mechanisms, and functions. Open Life Sciences. 2011; 6(5): 785-801, doi: 10.2478/s11535-011-0054-4.

47. Li Z, Zhang T, Dai H, et al. Involvement of endoplasmic reticulum stress in myocardial apoptosis of streptozocin-induced diabetic rats. J Clin Biochem Nutr. 2007; 41(1): 58-67, doi: 10.3164/ jcbn.2007008, indexed in Pubmed: 18392099.

48. Li Z, Zhang T, Dai H, et al. Endoplasmic reticulum stress is involved in myocardial apoptosis of streptozocin-induced diabetic rats. J Endocrinology. 2010; 207(1): 123, doi: 10.1677/joe-070230r.

49. Xu J, Zhou Qi, Xu W, et al. Endoplasmic reticulum stress and diabetic cardiomyopathy. Exp Diabetes Res. 2012; 2012 827971, doi: 10.1155/2012/827971, indexed in Pubmed: 22144992.

50. Liu J, Liu Yu, Chen Li, et al. Glucagon-like peptide-1 analog liraglutide protects against diabetic cardiomyopathy by the inhibition of the endoplasmic reticulum stress pathway. J Diabetes Res. 2013; 2013: 630537, doi: 10.1155/2013/630537, indexed in Pubmed: 23671882.

51. Howarth FC, Qureshi MA, Hassan Z, et al. Changing pattern of gene expression is associated with ventricular myocyte dysfunction and altered mechanisms of $\mathrm{Ca} 2+$ signalling in young type 2 Zucker diabetic fatty rat heart. Exp Physiol. 2011; 96(3): 325-337, doi: 10.1113/expphysiol.2010.055574, indexed in Pubmed: 21216827.

52. Shinozawa T, Imahashi K, Sawada $\mathrm{H}$, et al. Determination of appropriate stage of human-induced pluripotent stem cell-derived cardiomyocytes for drug screening and pharmacological evaluation in vitro. J Biomol Screen. 2012; 17(9): 1192-1203, doi: 10.1177/1087057112449864, indexed in Pubmed: 22706346 . 